\title{
Source Localization Technique for Multiple Acoustic Sources by Using Acoustic Beating Envelopes
}

\author{
Kyoung-Sik Choi*, Sung-Jae Heo, Tae-Wan Kim
}

Korea Aerospace Industries Ltd., Sacheon-City, Gyeongnam, 664-710, South Korea

Received: March 31, 2017; Accepted: April 11, 2017; Published: April 23, 2017

*Corresponding author: Kyoung-Sik Choi, Korea Aerospace Industries Ltd., Sacheon-City, Gyeongnam, 664-710, South Korea, E-mail: cks@koreaaero.com

\begin{abstract}
We propose here new technique to detect multiple acoustic source positions. This technique is more simple and cost saving method. We used several acoustic sensors to detect the exact positions of multiple acoustic sources simultaneously. Especially acoustic waves higher than audio frequency were used to avoid unnecessary acoustic noises. In this case, higher frequency means short wave length, which makes it difficult to extract the phase difference between two sensors when the distance of the two sensors exceed half of wave length. In this paper, we applied beating signal which is composed of two slightly different close frequencies. The wave length of the beating signal's envelope which is longer than those of the orignals is controllable. Using the envelope of beating signal gives us many advantages. For example, we are free from unnecessary acoustic noises because of using higher frequency than audio frequency. Additionally, the wave length of beating signal is under control which means sensor position is not limited for the signal processing. We applied here a hypothetical simulation to prove it. by some final considerations.
\end{abstract}

Keywords: Source localization; Beating signal; Envelope; Phase delay; Acoustic wave; TDOA;

\section{Introduction}

Source localization techniques have been proposed in many areas for the various applications [1-3]. We proposed here more simple and cost saving method. The main key idea is using beating signal which is composed of two slightly different close frequencies. Especially the frequencies of two waves are higher than audio frequency. The reason why we use high frequency is low frequency (audio frequency) yields unnecessary acoustic noises and most of the mechanical noises are ranging under audio frequency. Mechanical noises could yield interferences. Because of these two critical issues, we determined to use high frequency. But high frequency means short wave length which makes a difficulty to extract exact phase difference when the distance of the two sensors is exceeding half of wave length. In order to solve this problem we used beating signal. Beating signal is composed of two slightly different close signals [4]. These two close waves make beating signal as a result of interference. The wave length of a beating signal's envelope is longer than those of the originals. It means we can extract longer wave from high frequency waves without any transform. This gives us many advantages. For example, we are free from choosing frequency range. Even though when we have to deal with high frequency, we can extract longer wave from original signals without any transform. For these reasons we used beating signal to estimate multiple acoustic sources. In order to estimate source location we have to extract arrival time difference of the received sensor signals respectively. Time differences could be extracted from phase differences. In this paper, we extracted beating signal's phase difference from original signals. We showed how to find a beating signal frequencies and phases and how to extract phase differences among beating signals. Finally showed how these phase differences are used finding source locations.

\section{Fundamental Theories}

\section{Beating Signal}

Beating signal is composed of two slightly different close waves [4]. The wave length of a beating signal's envelope is longer than those of the originals. This allows us to use high frequency.

When we estimate source location by using phase differences of received signals, we would confront some limitations. The wave length should be two times or longer than the distance between two sensors. For the reason that a wave length of a beating signal's envelope is much longer than those of the originals, we could put the sensors far away each other. That means sensors' locations are free for the wave length because we are able to control wave length of a beating signal. We can make beating signal by using frequency mixer or simply summing slightly different two frequencies. In case of frequency mixer (Figure 1), put the high frequency as $\omega_{h}$ and low frequency as $\omega_{l}$ respectively. These two signals satisfy the conditions below.

$$
\omega_{h} \gg \omega_{l}\left(a b s\left(\omega_{h}-\omega_{l}\right)>20 K H z\right)
$$




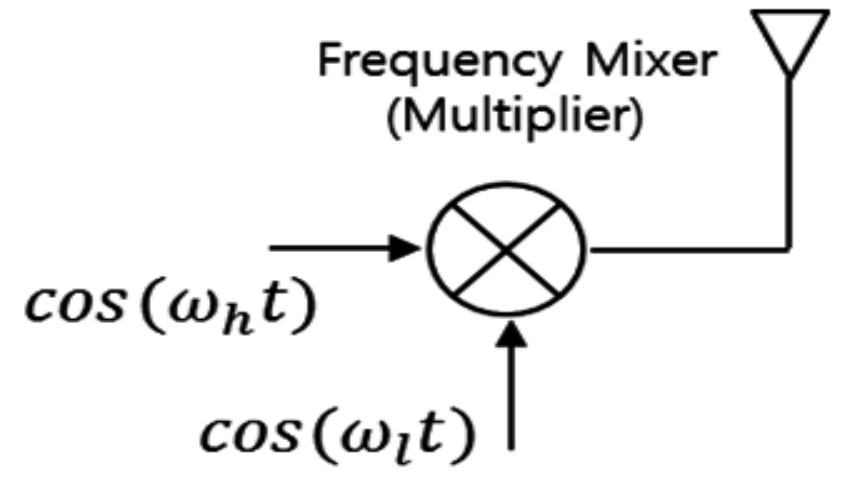

Figure 1: Frequency mixer can get beating signal

For the conditions above, $\omega_{h}$ should be much higher than $\omega_{l}$. Then we can make beating signal with these two frequencies by using frequency mixer. This can be expressed as below.

$\cos \left(\omega_{h} t\right) \times \cos \left(\omega_{l} t\right)=\frac{1}{2} \cos \left(\left(\omega_{h}+\omega_{l}\right) t\right)+\frac{1}{2} \cos \left(\left(\omega_{h}-\omega_{l}\right) t\right)$

In this case, each wave length of $\omega_{h}-\omega_{l}$ and $\omega_{h}+\omega_{l}$ are slightly different. As a result, these two frequencies make beating signal.

The other way to make beating signal is using two frequencies. This is a simpler way to make beating signal. That directly summates slightly different two waves $\left(\omega_{1}, \omega_{2}\right)$ We can assume that each wave $\left(\omega_{1}, \omega_{2}\right)$ is composed of two frequencies of $\omega_{h}, \omega_{l}$. These two frequencies might satisfy Eq.(1) Then we can express $\omega_{1}, \omega_{2}$ as below.

$$
\omega_{1}=\omega_{h}+\omega_{l}, \omega_{2}=\omega_{h}-\omega_{l}
$$

Then we can get slightly different two close waves . Figure 2 shows how to make beating signal.

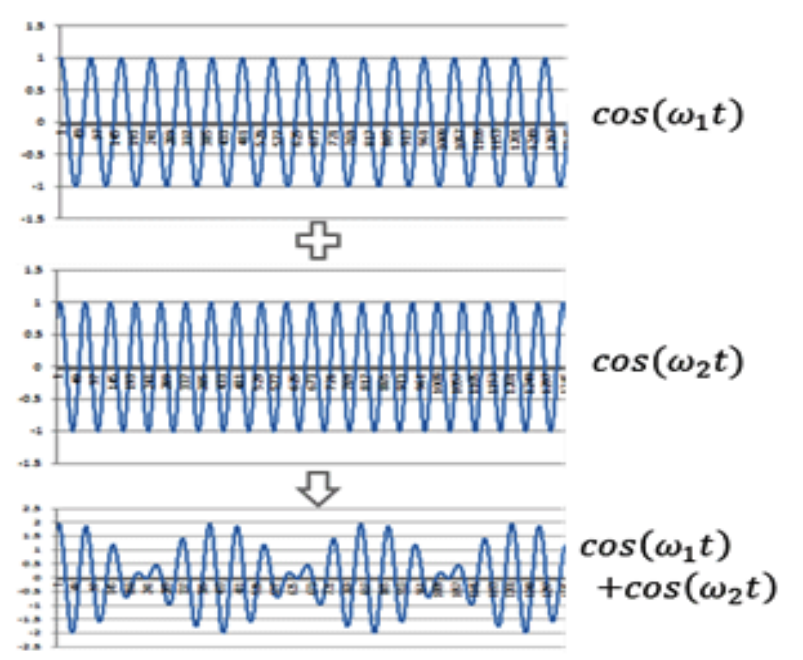

Figure2: One beating signal is composed of slightly different two close waves

\section{Envelope of Beating Signal}

The next step is extracting frequency and phase of a beating signal. One method of extracting the beating signal's envelope is to use the Hilbert Transform $[8,9]$. The Hilbert Transform of a real-valued time domain signal ${ }^{\wedge} \mathrm{X}(\mathrm{t})$ is another real-valued time domain signal denoted by $x(t)$, such that $z(t)=x(t)+j x(t)$ The Hilbert transform of $x(t)$ extending over the range $-\infty<t<\infty$ is a real-valued function $x(t)$ defined by

$$
\hat{x}(t)=H\{x(t)\}=\int_{-\infty}^{\infty} \frac{x(\tau)}{\pi(t-\tau)} d \tau
$$

$x(t)$ is the convolution integral of $x(t)$ and $1 / \pi t$,

$$
\hat{x}(t)=x(t) *(1 / \pi t)
$$

A useful way to compute the Hilbert Transform $x(t)$ of $x(t)$ is via the analytic signal $\mathrm{z}(\mathrm{t})=x(t)+\mathrm{j} x(t)$. One can also write $\mathrm{z}(\mathrm{t})=A(t) e^{j \theta(t)}$, where $\mathrm{A}(\mathrm{t})$ denotes the envelope signal of $x(t)$ and $\mathrm{e}(\mathrm{t})$ denotes the instantaneous phase signal of $x(t)$.In terms of $x(t)$ and $x(t)$, it is clear that

$$
\begin{aligned}
& A(t)=\left[x^{2}(t)+\hat{x}^{2}(t)\right]^{1 / 2} \\
& \theta(t)=\tan ^{-1}\left[\frac{\hat{x}(t)}{x(t)}\right]
\end{aligned}
$$

So, we can obtain envelopes by using Eq.(6).

We showed here how we can get beating signal's envelop by using Hilbert Transform. But in many cases, received signals have lots of noises and it is not always limited to one sources. If there are many sources then it is not easy to find beating signal's envelope. So we introduced here more general method to find frequency and phase of a beating signal. Let's put two frequencies as $\omega_{1}\left(=\omega_{h}+\omega_{l}\right), \omega_{2}\left(=\omega_{h}-\omega_{l}\right)$, and its correspoding phases $\varphi\left(\omega_{1}\right), \varphi\left(\omega_{2}\right)$ respectively. These two waves are satifying the conditions of Eq.(1) and Eq.(3). Then beating signal's frequency $\omega_{\text {beat }}$ And its corresponding phase $\varphi\left(\omega_{\text {beat }}\right)$ can be expressed as below

$$
\begin{aligned}
& \omega_{\text {beat }}= \omega_{1}-\omega_{2}\left(\omega_{1}>\omega_{2}\right) \\
& \varphi_{\text {beat }}=\varphi\left(\omega_{1}\right)-\varphi\left(\omega_{2}\right)
\end{aligned}
$$

We can express a beating sginal from Eq.(2) as below 
$\cos \left(\omega_{h}\left(t-\tau_{h}\right)\right) \times \cos \left(\omega_{l}\left(t-\tau_{l}\right)\right)=1 / 2 \cos \left(\left(\omega_{h}+\omega_{l}\right) t-\left(\omega_{h} \tau_{h}+\omega_{l} \tau_{l}\right)\right)+1 / 2 \cos \left(\left(\omega_{h}-\omega_{l}\right) t-\left(\omega_{h} \tau_{h}-\omega_{l} \tau_{l}\right)\right)$

Then we can extract beating signal's frequency and phase using Eq.(7) and Eq.(8). (Figure 3 shows beating signal and it's envelope)

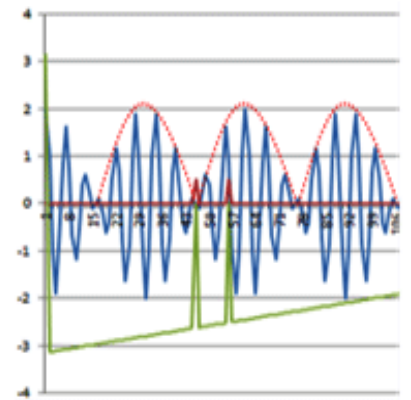

(a)

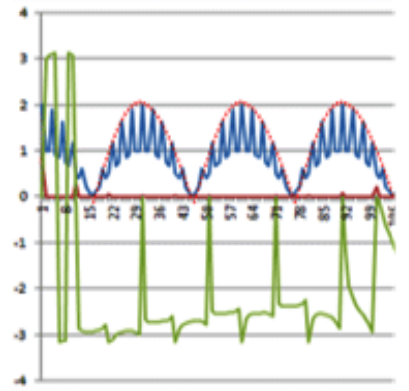

(b)
Figure 3: Envelope of beating signal (a) beating signal, (b) envelope for the beating signaleating signal

$$
\begin{gathered}
\omega_{\text {beating }}=\omega_{1}-\omega_{2}=2 \omega_{l} \\
\varphi_{\text {beating }}=\varphi\left(\omega_{1}\right)-\varphi\left(\omega_{2}\right)=2 \omega_{l} \tau_{l}
\end{gathered}
$$

\section{Frequency and Phase of a Beating Signal}

In order to estimate an acoustic source locations, the time delays between acoutic sensors signals must be calculated. The time delay is related to the phase delay of the envelopes. But as shown in Figure 4,

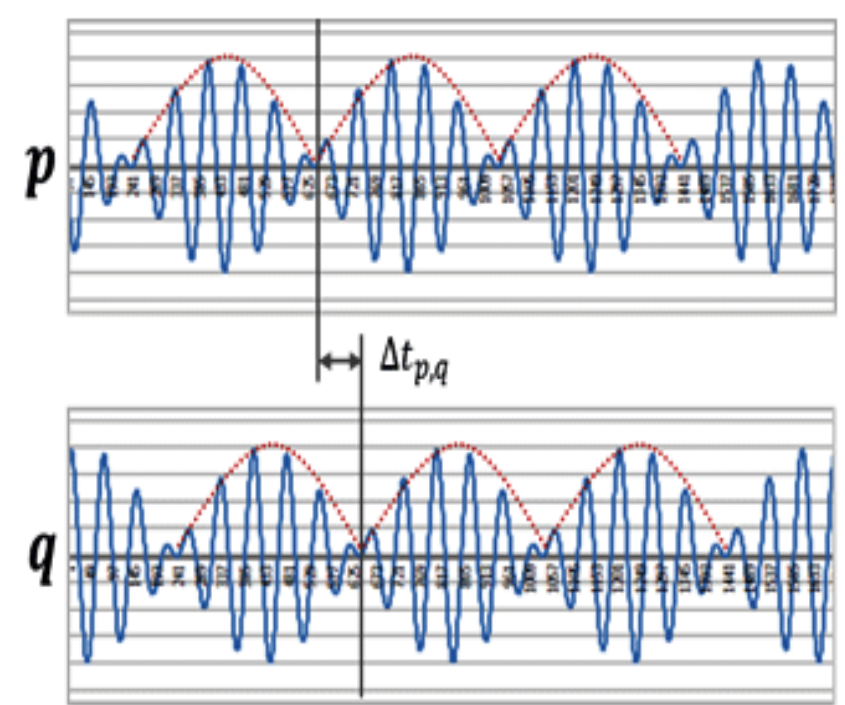

Figure 4: Time delay of a beating signal's envelope it is difficult to find the arrival time difference using the envelope in the time domain. So, we tried to find the time delays using the envelope's phase delay in the frequency domain [7-9]. We are dealing with two beating signals $p(t)$ and $q(t)$. We could think

$p(t)$ and $q(t)$. are received signals at a different two sensors, and $\tau_{d}$ means the time delay between $p(t)$ and $q(t)$. Then we can express it as

$p(t)=A\left\{\cos \left(\omega_{1} t-\varphi_{1}\right)+\cos \left(\omega_{2} t-\varphi_{2}\right)\right\}$

In this case, the frequency and phase of beating signal are $\omega_{1}-\omega_{2}\left(\omega_{1}>\omega_{2}\right), \varphi_{1}-\varphi_{2}$ respectively.

Then,

$$
\begin{aligned}
& q(t)=a \times p\left(t-t_{d}\right) \\
& =a \times A\left\{\cos \left(w_{1}\left(t-t_{d}\right)-j_{1}\right)+\cos \left(w_{2}\left(t-t_{d}\right)-j_{2}\right)\right\}
\end{aligned}
$$

where $\omega_{1}, \omega_{2}$ are the frequency components composing beating signal and $\alpha$ is the magnitude ratio between $p(t)$ and $q(t)$. The cross-correlation of Eqs. (10) and (11) is derived as the following equation.

$R_{p, q}(\tau)=\alpha E[p(t) q(t+\tau)]=\alpha R_{p, p}\left(\tau-\tau_{d}\right)$

where $\mathrm{E}[\mathrm{a}]$ is the expected value of a. This equation shows that the cross-correlation of two signals can be expressed as the autocorrelation that has a time delay $\tau_{d}$. If Eq. (12) is transformed to the frequency domain, we can obtain the cross-spectrum. Let

$P(\omega)$ and $\mathrm{Q}(\omega)$ denote the components of the Fourier transform of $p(t)$ and $q(t)$; the cross-spectrum is

$$
\begin{aligned}
& S_{p, q}(\omega)=P^{*}(\omega) \times Q(\omega) \\
& =|P(w)||Q(w)| e^{j\left\{j_{p}(w)-j_{q}(w)\right\}}
\end{aligned}
$$

where $\varphi_{p}(\omega)$ and $\varphi_{q}(\omega)$ mean the phase of $p(t)$ and $q(t)$. respectively. Figure 4 shows two beating signals having some phase differences.

\section{TDOA(Time Difference of Arrival)}

In order to extract Time-Difference-Of-Arrival (TDOA) between two received beating signals. We should compare the arrived time difference of the two received beating signals. Put received beating signal's phases as $\varphi_{p}, \varphi_{q}$ respectively then phase difference between two beating signals could be expressed as

$$
\begin{aligned}
& \varphi_{p}-\varphi_{q}= \\
& \varphi_{p}\left(\omega_{1}\right)-\varphi_{p}\left(\omega_{2}\right)-\left(\varphi_{q}\left(\omega_{1}\right)-\varphi_{q}\left(\omega_{2}\right)\right)= \\
& \varphi_{p}\left(\omega_{1}\right)-\varphi_{q}\left(\omega_{1}\right)-\left(\varphi_{p}\left(\omega_{2}\right)-\varphi_{q}\left(\omega_{2}\right)\right)
\end{aligned}
$$

Here, we could get some conclusion. Phase difference between two beating signals is a subtraction of a same frequency. 
This means we can get beating signal's phase differences by crossing spectrum. It is not necessary to find beating signal envelopes. The relationship between period ' $\mathrm{T}$ ' and frequency ' $f$ ' is $\mathrm{T}=1 / f$. The time delay of acoustic beating envelope $\Delta t_{p, q}(f)$ can be obtained by multiplying the period by the ratio of phase delay. Therefore, the time delay can be expressed as following equation.

$$
\Delta t_{p, q}(f)=\frac{1}{f} \times \frac{\varphi_{p}(f)-\varphi_{q}(f)}{2 \pi}
$$

The distance difference transferred from the impact source to the two sensors is derived by multiplying Eq.(11) by the sound velocity. v=340m/s, @ $15^{\circ} \mathrm{C}$

$$
\Delta r_{p, q}=r_{p}-r_{q}=v \times \Delta t_{p, q}(f)
$$

\section{Triangular Method}

Triangulation method was applied to point out an exact position. Figure 5 shows the triangular method for only two sensors. And figure 6 shows for more than three sensors.

In figure 7 Triangulation method looks for the hyperbola intersection point based on time-of-arrival differences (TOADs) of a burst between three different sensors $[3,5,6]$. Assume sensor number is $\mathrm{N}$, then we can get hyperbola lines number of

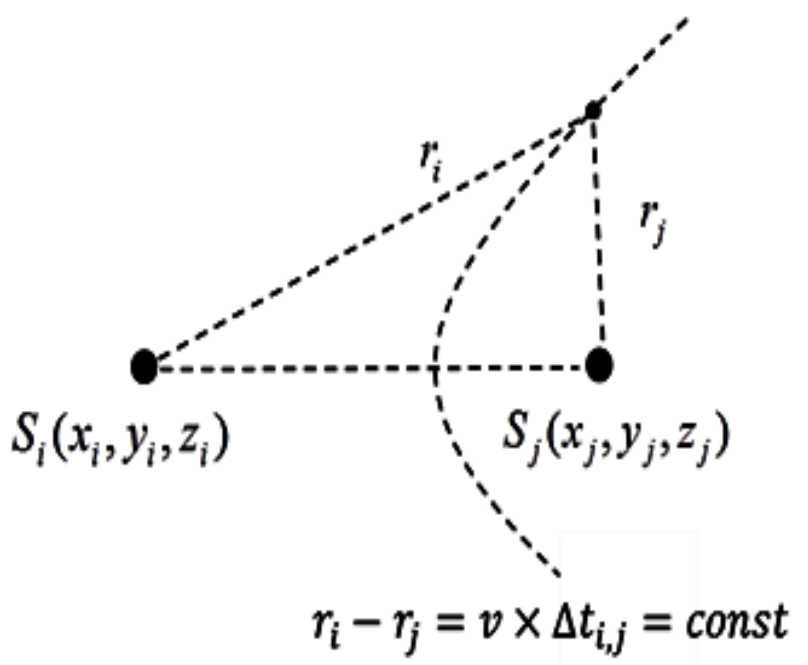

Figure 5: Hyperbola line when only two sensors are used

$$
M=\frac{N !}{2 !(N-2) !}=\frac{N(N-1)}{2}
$$

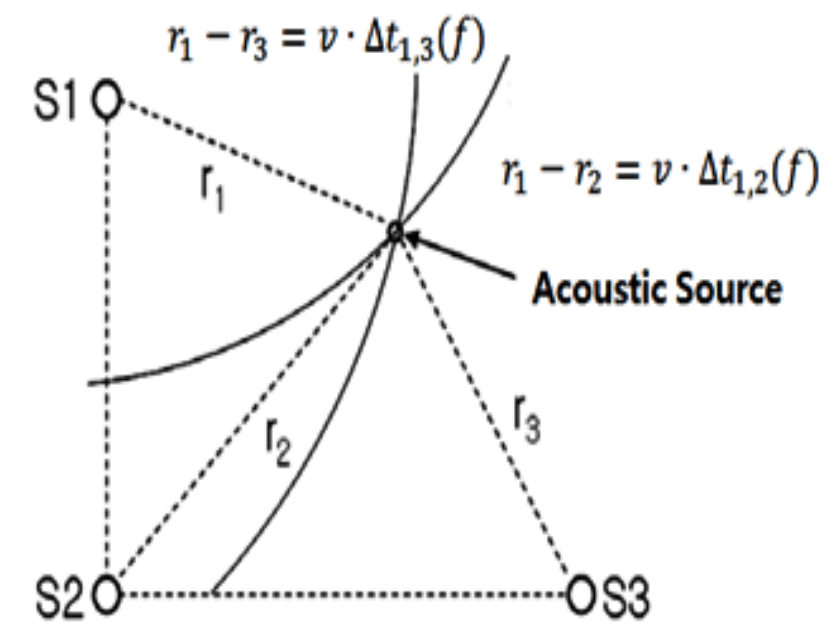

Figure 6: Hyperbola line when more than 3 sensors are used

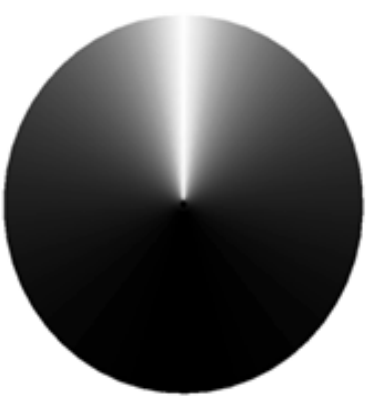

(a)

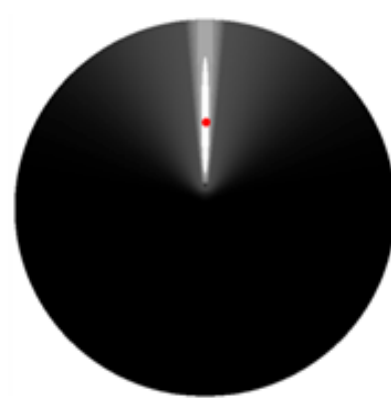

(b)
Figure 7: Sample of software simulation for triangulation method (a) only two sensors are used, (b) more than 3 sensors are used

\section{Simulation and Verification}

In order to verify our proposed theory by experiment, we put two acoustic emission sources and three sensors. Figure 8 shows the conditions of a hypothetical simulation

The coordinates of two acoustic emission sources are E1 $(250,350)$ and E2 $(350,250)$. The coordinates of three acoustic sensors are $S 1(0,0), S 2(600,0)$ and $S 3(300,600)$ respectively. We can derive two beating signals from the frequencies of the two acoustic emission sources. The first beating signal is composed of $25 \mathrm{KHz}, 25.5 \mathrm{KHz}$, and the second beating signal is composed of $27 \mathrm{KHz}$ and $27.5 \mathrm{KHz}$. Figure 9 shows received signals from S1, S2 and S3. Figure 10 shows the result of cross power spectrum of the received signals. In order to find phase differences between beating signals depending on Eq.(14) we should find phase differences of the same frequencies first. The phase differences of the same frequencies for the first beating signal (E1) are as below 


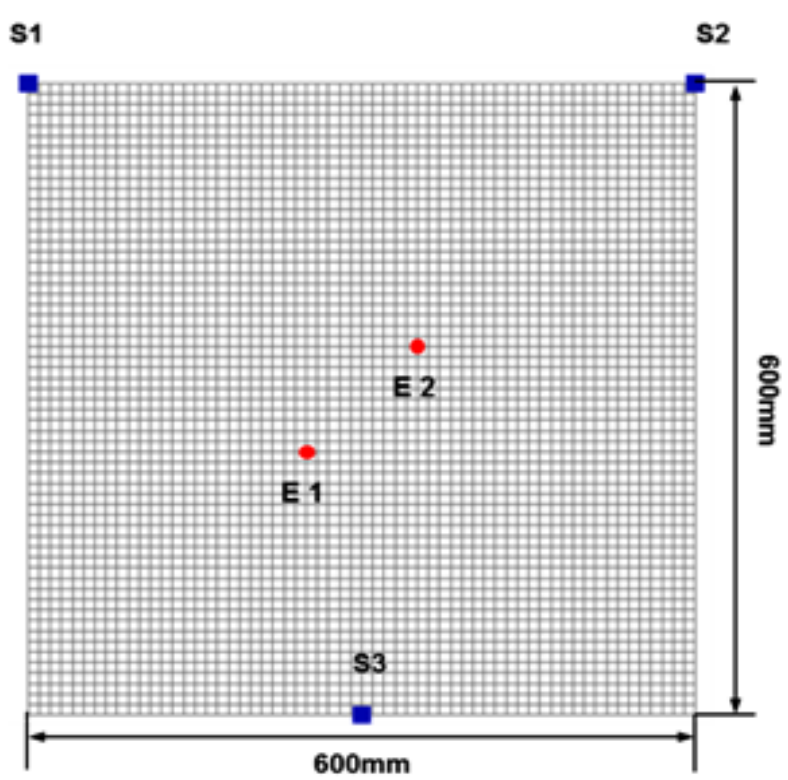

Figure 8: Simulation conditions which motion box area is $600 \mathrm{~mm} \mathrm{x}$ $600 \mathrm{~mm}$, red circle denotes the emission sources

(a)

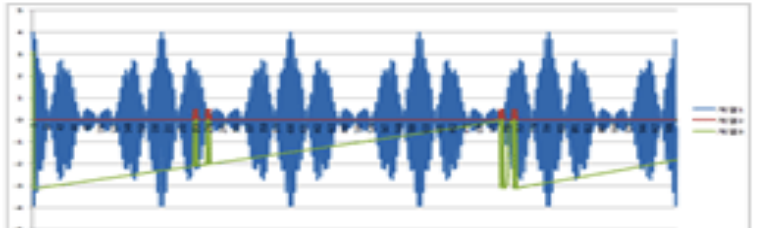

(b)

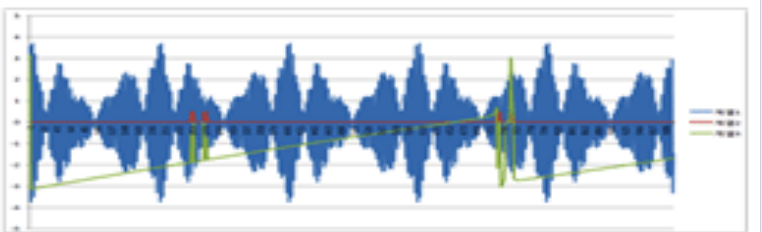

(c)

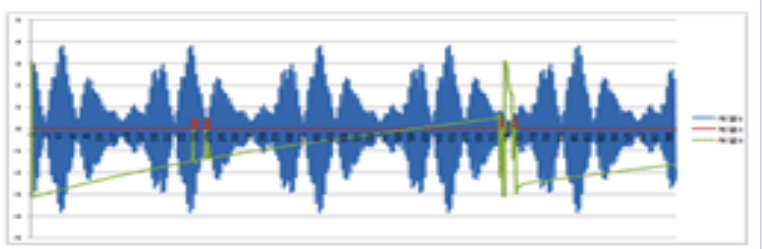

Figure 9: Measured signals for each acoustic sensors, (a) S1, (b) S2, (c) S3 (a)

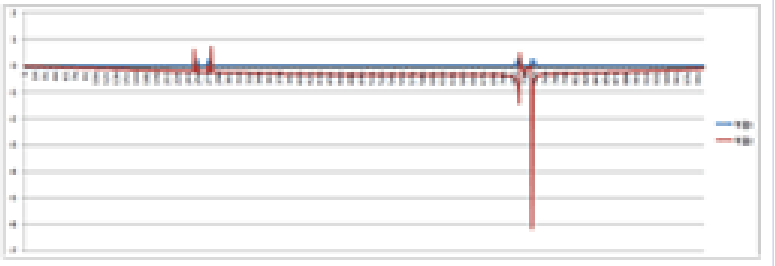

(b)

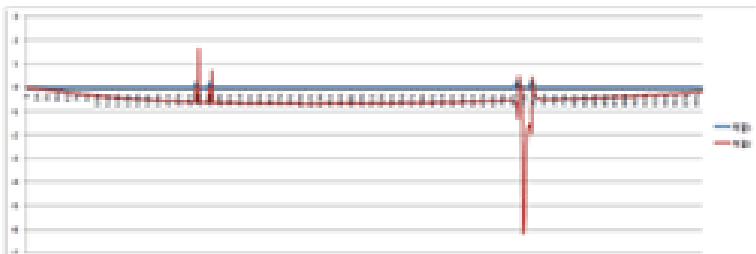

(c)

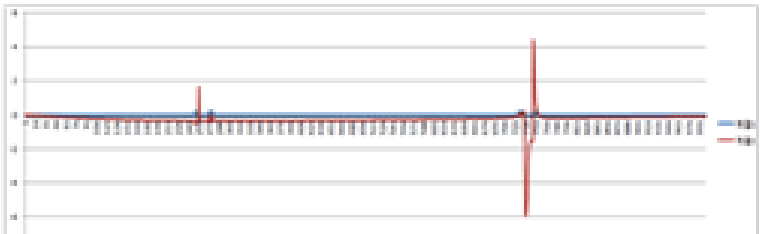

Figure 10: The result of cross power spectrum (a) S1\&S2 (b) S1\&S3, (c) S2\&S3. Blue line denote magnitude, red line denote phase

$K H z)=0.594$,

$\varphi_{1,3}(25 \mathrm{KHz})=0.0$,

$\varphi_{2,3}(25 \mathrm{KHz})=-0.594$,

$\varphi_{1,2}(25.5 \mathrm{KHz})=0.0$,

$\varphi_{1,3}(25.5 \mathrm{KHz})=0.0$,

$\varphi_{2,3}(25.5 \mathrm{KHz})=1.615$

and then we can extract phase difference for the two beating signals by using Eq.(14)

$$
\begin{aligned}
& \varphi_{1,2}(25.5 \mathrm{KHz})-\varphi_{1,2}(25 \mathrm{KHz})=-0.594 \\
& \varphi_{1,3}(25.5 \mathrm{KHz})-\varphi_{1,3}(25 \mathrm{KHz})=1.615 \\
& \varphi_{2,3}(25.5 \mathrm{~Hz})-\varphi_{2,3}(25 \mathrm{KHz})=2.209
\end{aligned}
$$
below

$$
\begin{aligned}
& \Delta t_{1,2}(0.5 \mathrm{KHz})=-0.000189 \\
& \Delta t_{1,3}(0.5 \mathrm{KHz})=0.000514
\end{aligned}
$$


$\Delta t_{2,3}(0.5 \mathrm{KHz})=0.000703$

Then the delay distance can be obtained by multiplying sound velocity.

$$
\begin{aligned}
\Delta r_{1,2} & =-0.06426 \mathrm{~m} \\
\Delta r_{1,3} & =0.17476 \mathrm{~m} \\
\Delta r_{2,3} & =0.23902 \mathrm{~m}
\end{aligned}
$$

The second beating signals are as below(E2)

$$
\begin{aligned}
& \varphi_{1,2}(27 \mathrm{KHz})=0.0 \\
& \varphi_{1,3}(27 \mathrm{KHz})=0.0 \\
& \varphi_{2,3}(27 \mathrm{KHz})=0.0 \\
& \varphi_{1,2}(27.5 \mathrm{KHz})=0.701 \\
& \varphi_{1,3}(27.5 \mathrm{KHz})=0.713 \\
& \varphi_{2,3}(27.5 \mathrm{KHz})=0.021
\end{aligned}
$$

For the second beating signal

$$
\begin{gathered}
\varphi_{1,2}(27,500 H z)-\varphi_{1,2}(27,000 H z)=0.701 \\
\varphi_{1,3}(27,500 H z)-\varphi_{1,3}(27,000 H z)=0.713 \\
\varphi_{2,3}(27,500 H z)-\varphi_{2,3}(27,000 H z)=0.012
\end{gathered}
$$

time delays for the second beating signal is

$$
\begin{gathered}
\Delta t_{1,2}(0.5 \mathrm{KHz})=0.000223 \\
\Delta t_{1,3}(0.5 \mathrm{KHz})=0.000227 \\
\Delta t_{2,3}(0.5 \mathrm{KHz})=0.000004
\end{gathered}
$$

Then the delay distance can be obtained by multiplying sound velocity.

$$
\begin{aligned}
& \Delta r_{1,2}=0.07582 \mathrm{~m} \\
& \Delta r_{1,3}=0.07718 \mathrm{~m} \\
& \Delta r_{2,3}=0.00136 \mathrm{~m}
\end{aligned}
$$

Figure 11 shows the hypothetical simulation result. We were able to verify our proposal. This is one of the best solutions to find exact source location for the two acoustic beating signals or more.

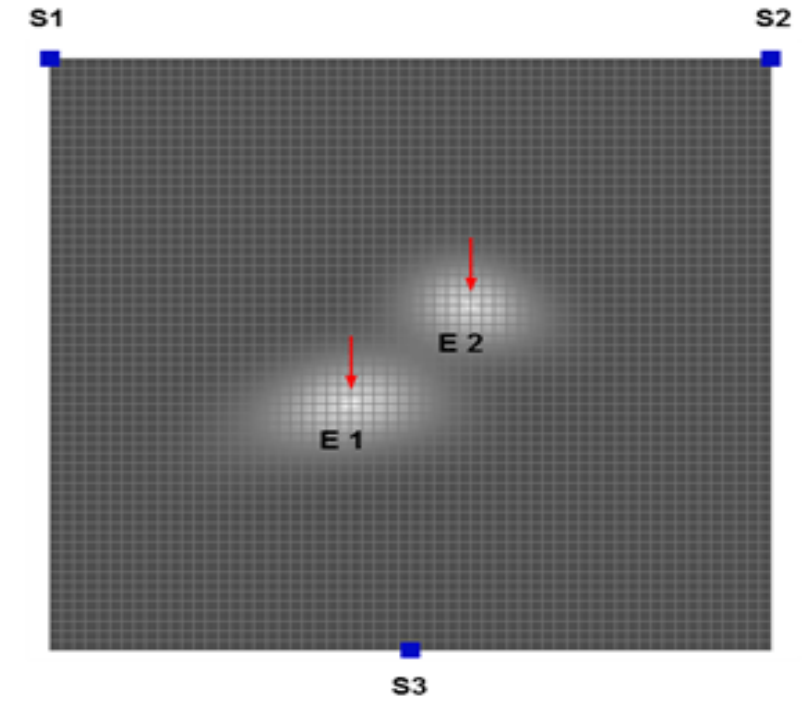

Figure 11: Source localization test result

\section{Conclusion}

We proposed here a localization technique for multiple acoustic sources using acoustic beating envelopes of which wave length are longer than audio frequency. Then we used a hypothetical simulation to prove it. The reason why we used high frequencies is not to make unnecessary acoustic noises. Additional purpose for using high frequency is to avoid unnecessary mechanical noise interferences. Because most mechanical noises are ranging under $20 \mathrm{KHz}$. We also newly proposed here how to use beating signal's envelope. Wave length of a beating signal is much longer than those of the originals. For this reason we are free from sensor locations. We were able to get several advantages by using acoustic beating signal.

\section{References}

1. Lubin BT, Longo R, Hammel T. Analysis of internals vibration monitoring systems data related to the St. Lucie 1 thermal shield failure. Progress in Nuclear Energy. 1988;21:117-126.

2. Olma BJ.Source location and mass estimation in loose parts monitoring of LWRs. Progress in Nuclear Energy. 1985;15:583-594.

3. Choi KS, Choi YC, Park JH, Kim HW. Source Localization Technique for Metallic Impact Source by Using Phase Delay between Different Type Sensors. KSNVE. 2008;18(11):1143-1149. DOI : 10.5050/ KSNVN.2008.18.11.1143

4. Lee HC. Robust Damage Diagnostic Method Using Short Time Fourier Transform and Beating. KSNVE. 2005;15(9):1108-1117. DOI : 10.5050/KSNVN.2005.15.9.1108

5. Kryter RC, Shahrokhi F. Summary of studies on methods for detecting, locating, and characterizing metallic loose parts in nuclear reactor coolant system. US nuclear regulatory commission report NUREG/CR2344. 1981. 
6. Choi KS, Kim JP, Won HK, Park JH, Kim IG. Source Localization Technique for Radar Pulse Emission by Using Scanning Method of Interest Area. KSAS. 2011;39(9):889-895.

7. Cohen L. Time-frequency Distribution. Prentice Hall PTR, Engle-wood Cliffs. New Jersey. 1995.

8. Randall RB. Frequency Analysis. 3rd Edition. 1987:19-31:58-62.

9. Julius SB, Allan GP. RANDOM DATA, Analysis and Measurement Procedures. 2nd Edition. 1986;109-159. 